\title{
Historique de l'évolution anthropique et dynamique du couvert végétal dans la commune de Bantè au Bénin
}

\author{
K. Innocent $\mathrm{AKOBI}^{1 *}$, Ernest $\mathrm{AMOUSSOU}^{2}$, Ibouraima $\mathrm{YABI}^{1}$ et Michel BOKO${ }^{1}$ \\ ${ }^{I}$ Département de Géographie et Aménagement du Territoire de l'Université d'Abomey Calavi (UAC), \\ Laboratoire Pierre PAGNEY, Abomey Calavi, Bénin. \\ ${ }^{2}$ Département de Géographie et Aménagement du Territoire de l'Université de Parakou (UP) Parakou, Bénin. \\ "Auteur correspondant ; E-mail: akobinno@yahoo.fr, Tel : (+229) 97485510
}

\section{RESUME}

Le couvert végétal de la commune de Bantè subit de forte pression anthropique. L'objectif visé a été d'analyser l'influence de la pression humaine sur l'évolution spatio-temporelle des unités paysagiques. Les données utilisées concernent les statistiques démographiques de l'INSAE des années1979, 1992, 2002 et 2013 , l'historique de l'installation des populations dans le temps et dans l'espace, les cartes planimétriques et les images Landsat TM de 1986, Landsat ETM+ de 2000 et Landsat OLI-TIRS de 2016 de la commune de Bantè. La cartographie des unités d'occupation des terres sur la base des données de la télédétection et leur étude diachronique ont été les principales méthodes utilisées. La cartographie des changements spatio-temporels du couvert végétal a révélé que la superficie des formations végétales naturelles est passée de 93,63\% dans la Commune en 1986 à 92,92\% en 2000 et à 38,83\% en 2016 au profit des champs et des jachères dont la superficie est passée de 6,19\% en 1986 à 6,68\% en 2000 et à 33,15\% en 2016. Entre ces trois dates, les formations saxicoles ont complètement disparu au profit des mosaïques de champs et jachères. Dans le même temps, le phénomène de colonisation agricole s'est accru avec la création de nombreux campements peuhls et fermes faisant passer le nombre de 100 à près de 200 lieux habités. En somme, la pression humaine sur les terres a engendré une dégradation des formations naturelles au profit des formations anthropiques, nécessitant ainsi, une gestion rationnelle de l'espace communal par les autorités locales en charge.

(C) 2018 International Formulae Group. All rights reserved.

Mots clés : Bénin, Bantè, pression anthropique, couvert végétal, peuplement, migration.

\section{History of anthropic and dynamic evolution of land covert in the district of Bantè in Benin}

\begin{abstract}
The vegetation cover of the Commune of Bantè is subjected to strong anthropogenic pressure. The objective was to analyze the influence of human pressure on the spatio-temporal evolution of land cover. The data used concern the INSAE population statistics for the years 1979, 1992, 2002 and 2013, the history of the installation of the populations in time and space, the planimetric maps and the Landsat TM images of 1986, Landsat ETM + 2000 and Landsat OLI-TIRS 2016 from the municipality of Bantè. Mapping land-use units on the basis of remote sensing data and their diachronic study were the main methods used. Mapping of spatio-
\end{abstract}


temporal changes in the land cover revealed that the area of natural plant formations increased from $93.63 \%$ in the Commune in 1986 to $92.92 \%$ in 2000 and to $38.83 \%$ in 2016 in favor of the fields and fallow land, which increased from $6.19 \%$ in 1986 to $6.68 \%$ in 2000 and to $33.15 \%$ in 2016 . Between these three dates, the saxicolic formations have completely disappeared in favor of mosaics of fields and fallow land. At the same time, the phenomenon of agricultural colonization has increased with the creation of numerous farms and camps, increasing the number from 100 to almost 200 inhabited places. In sum, the human pressure on the lands has caused a degradation of natural formations in favor of human formations, thus requiring a rational management of the communal space by the local authorities in charge.

(C) 2018 International Formulae Group. All rights reserved.

Keywords: Benin, Bantè, Anthropic pressure, land cover, settlement, migration.

\section{INTRODUCTION}

Dans les pays africains, la tendance à la dégradation des ressources forestières du fait des activités humaines est inquiétante (Anthelme et al, 2006; Amani et Touré, 2015 ; Milogo et al., 2017). Au Bénin, elle n'est pas moins inquiétante dans la mesure où le couvert végétal dans toutes les régions est en constante régression en raison des facteurs anthropiques: activités agropastorales, exploitation du bois, implantations d'établissements humains, etc. (Oloukoi et al, 2006; Tenté et al., 2011; Houessou et al., 2013 ; Sitondji et al., 2013; Avakoudjo et al., 2014). Ainsi, le taux de dégradation du couvert végétal à l'échelle nationale est estimé en 2005 par le FAO à 70.000 hectares par an (Arouna, 2012). Ce taux national de recul du couvert végétal n'est pas sans cacher des disparités régionales et même locales. Or, la végétation est l'une des composantes majeures de l'environnement dont la sauvegarde constitue, sans nul doute, un enjeu planétaire depuis quelques décennies. En effet, la végétation conditionne en grande partie l'état des sols, de la faune et d'autres paramètres de l'existence des êtres vivants dont l'homme son prédateur. De même, dans le cadre de la lutte contre les effets des changements climatiques, la préservation du couvert végétal et le reboisement sont considérés comme une méthode à encourager dans les pays en développement comme le Bénin (Niassé et al., 2004; Boko et al., 2007; PANA-Bénin, 2008).

Dans le contexte actuel de la décentralisation, l'étude de la pression de l'homme sur le couvert végétal à l'échelle des Communes paraît très opportune car les questions d'affectation et de désaffectation des terres qui ont en fait des impacts majeurs sur la flore et la faune sont de la compétence des Communes (RB, 1999). Il serait alors judicieux d'étudier l'évolution du couvert végétal sur les enjeux du développement local durable au regard des prérogatives conférées aux autorités locales en matière d'aménagement et du développement local (RB, 1999). L'objectif de cette étude était d'analyser l'évolution des unités paysagères dans la commune de Bantè au regard de la poussée démographique et de l'expansion des colons agricoles qui induisent la prolifération des agglomérations.

\section{MATERIEL ET METHODES \\ Présentation géographique du cadre d'étude}

Située entre $8^{\circ} 4^{\prime}$ et $8^{\circ} 37^{\prime} \mathrm{N}$ et $1^{\circ} 36^{\prime}$ et $2^{\circ} 11^{\prime} \mathrm{E}$ (Figure 1 ), la Commune de Bantè est créée en 1978 comme district rural et compte actuellement 9 arrondissements. Elle est située au centre du Bénin et plus précisément au Nord-ouest du Département des Collines. Elle est limitée au Nord par le Département de la Donga, au Sud par la Commune de Savalou, à l'Est par la Commune de Glazoué et à l'Ouest par la République du Togo. Elle couvre une superficie de $2695 \mathrm{~km}^{2}$ et abrite une population dénombrée à 107181 habitants (INSAE, 2016) soit une densité moyenne de $39 \mathrm{hts} / \mathrm{km}^{2}$. 


\section{Données utilisées}

Il s'agit des données sociodémographiques et planimétriques. Les données socio-démographiques concernent les statistiques démographiques de l'INSAE des années1979, 1992, 2002 et 2013 et l'historique de l'installation des populations dans le temps et dans l'espace. Les données planimétriques utilisées sont: la carte topographique de la commune de Bantè, les images Landsat TM de 1986, Landsat ETM+ de 2000 et Landsat OLI-TIRS de 2016. Ces différentes images ont été téléchargées sur le site de earthexplorer.usgs.gov au format GEOTIFF.

\section{Matériel et outils utilisés}

Les enquêtes de terrain ont été réalisées à l'aide de plusieurs outils, notamment un questionnaire à l'endroit de la population cible, un guide d'entretien pour collecter des informations auprès des personnes ressources, une fiche de relevé des coordonnées géographiques, un GPS (Global Positioning System) pour la collecte des coordonnées géographiques des localités et équipements scolaires, le logiciel ArcGIS pour la réalisation des cartes et le logiciel SPSS pour le traitement des données sociodémographiques.

\section{Travaux de terrain}

Ils regroupent les observations, les enquêtes en milieu réel, la prise de différentes mesures. Les observations directes ont consisté à la contemplation des phénomènes et à la découverte des faits qui nous paraissaient illustratifs des témoignages reçus dans les fermes et hameaux visités. Les enquêtes ont été orientées vers les groupes cibles avec un échantillonnage par groupe socioprofessionnel dans une proportion variable. Il s'agit des élus communaux et locaux (10\%) des agents de la mairie (5\%), des exploitants forestiers (10\%), des producteurs (55\%), des agents de la section communale de développement agricole (5\%) des charbonniers (10\%), scieurs (3\%) des agents des eaux et forêts (2\%). Au total, 400 personnes ont été interrogées par questionnaire. L'entretien par focus group a été aussi réalisé auprès des populations de 27 localités à raison de 1 village administratif et 2 hameaux par arrondissement. L'enquête historique a consisté à recenser systématiquement tous les lieux habités et à dater la période de création de chacun d'eux afin de retracer le processus du peuplement du territoire. A cet effet, les vieux chefs coutumiers témoins directs ou indirects de la création des nouveaux villages et hameaux ont été consultés au travers des discussions et échanges. Les différentes informations ont été croisées et triangulées. Les informations collectées ont permis d'établir un lien entre l'évolution du peuplement humain et l'évolution du couvert végétal.

\section{Traitement et analyse des données}

Ils ont consisté à la réalisation en série des cartes de peuplement mettant en relief le semi continu dans le temps et dans l'espace des lieux habités. La carte de situation géographique a été corrigée sur la base des réalités observées sur le terrain par rapport à la localisation des établissements humains. Les lieux habités ont été géoréférencés et classés selon leur période de création pour montrer la dynamique du peuplement. De même, la cartographie de la dynamique de l'occupation du sol a été analysée à travers les cartes de végétation de 1986, de 2000 et de 2016. L'évolution des formations végétales des autres unités d'occupation du sol entre ces trois dates a été ensuite évaluée à travers les taux globaux d'évolution, la matrice de transition et les taux de conversion.

La matrice de transition a permis de mettre en évidence les différentes formes de conversions qu'ont subies les unités paysagères entre 1986, 2000 et 2016. Elle est constituée de $\mathrm{x}$ lignes et de y colonnes. Le nombre de lignes de la matrice indique le nombre de formations végétales au temps t0 tandis que le nombre de colonnes de la matrice indique le nombre d'unités paysagères converties au temps $\mathrm{t} 1$.

Le taux de conversion d'une classe d'unité d'occupation du sol correspond au 
degré de transformation subie par cette classe en se convertissant vers d'autres classes (Arouna, 2012; Toko Issiaka et al., 2016). C'est donc la quantité de changements observés au niveau d'une unité d'occupation du sol entre 2000 et 2013. Il permettra ainsi de mesurer le degré de conversion d'une unité donnée en d'autres unités d'occupation du sol. Il s'obtiendra à partir de la matrice de transition suivant la formule:

$T_{c}=\frac{S_{i t}-S_{i s}}{S_{i t}} \times 100$
$\mathrm{T}_{\mathrm{c}}$ : Taux de conversion; $\mathrm{S}_{i t}$ : Superficie de l'unité paysagère $i$ à la date initiale $\mathrm{t} ; \mathrm{S}_{i s}$ : Superficie de la même unité demeurée stable à la date $t_{1}$.

Pour mieux appréhender les problèmes que soulève ce sujet et en faire une bonne interprétation, le modèle d'analyse FPEIR (Force Motrice, Pression, Etat, Impact et Résultat) a été adoptée pour une meilleure réponse aux problèmes environnementaux dans la commune.

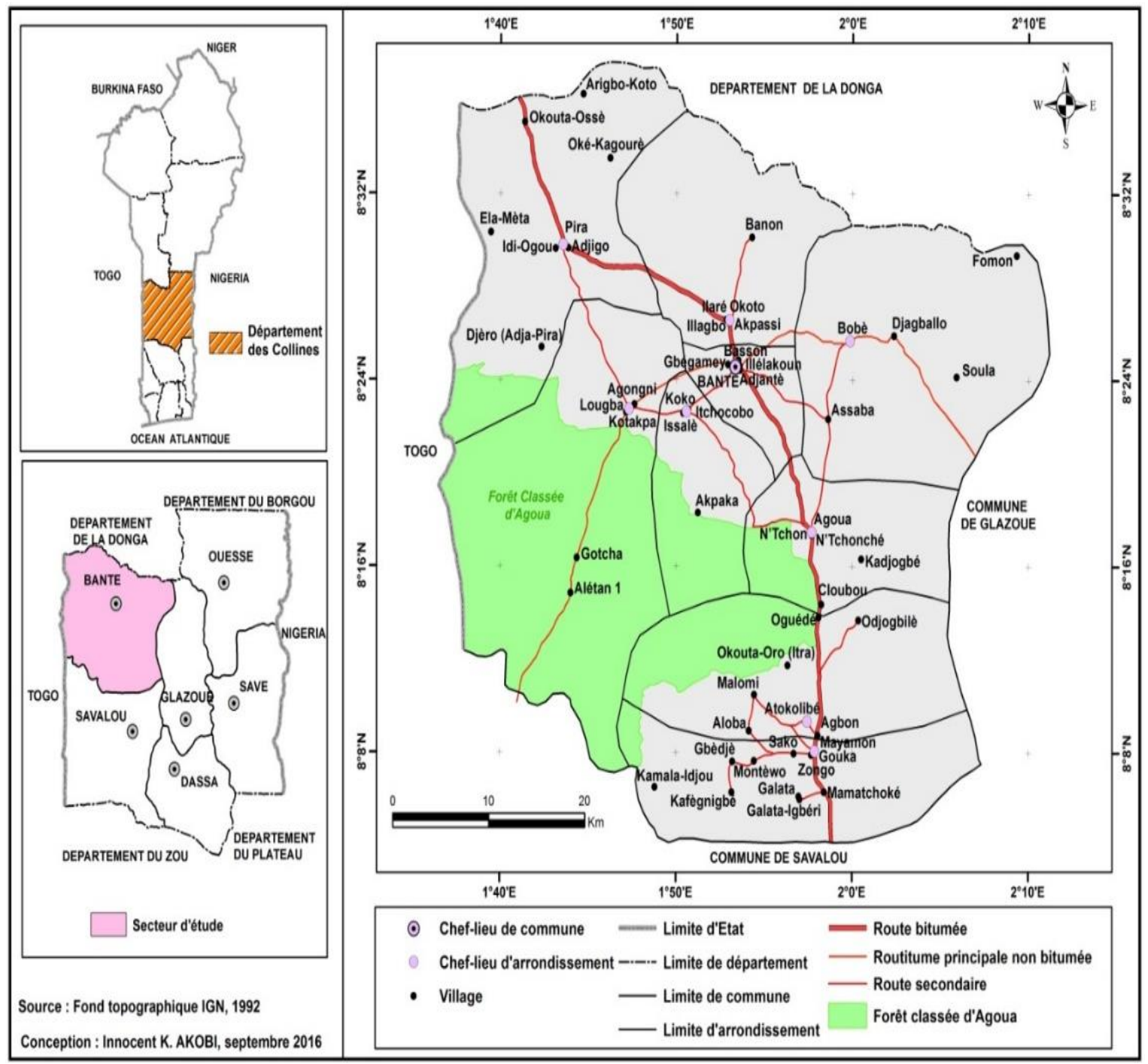

Figure 1: Situation géographique de la Commune de Bantè. 


\section{RESULTATS}

L'emprise de l'homme sur le couvert végétal est mesurée à travers ses activités qu'il exerce en aménageant son milieu. Qu'il s'agisse de l'habitat, des champs, des routes, des aires de pâturage, chacun de ces éléments influence d'une manière ou d'une autre le couvert végétal. C'est pourquoi, il importe de présenter d'abord l'évolution spatiotemporelle du peuplement avant d'étudier l'évolution spatio-temporelle du couvert végétal qui est largement tributaire de la pression anthropique.

\section{Pression anthropique à travers la dynamique du peuplement et l'exploitation des ressources naturelles}

D'après les enquêtes de terrain, on peut découper le long processus de peuplement du territoire de l'actuelle Commune de Bantè en trois grandes périodes: les périodes précoloniale, coloniale et postcoloniale. Suivant ce découpage, les lieux habités ont été regroupés selon leur période de création (Figure 2).

La période précoloniale, la plus longue, est celle de l'installation des peuples Isha et Ifè venus respectivement de «Ilé-Sha » et «Ilé-Ifè » de l'actuel Nigéria, vers le XII ${ }^{\mathrm{e}}$ siècle. C'est une période très mouvementée puisqu'elle est marquée par les guerres tribales et les razzias esclavagistes. L'insécurité caractéristique de cette période a favorisé le peuplement à habitat groupé des autochtones. Les premiers lieux habités sont Adjantè, Lougba, Koko, Lougba, Banon, Akpassi, Djagbalo, Bobè, pour les Isha au Nord et les localités d'Ilodji que sont Gouka, Mamatchoké, Galata, Mayamon, Agbon, Atokolibé, Malomi, Aloba et Sako pour les Ifè au Sud.

La période coloniale marquée par la pénétration et l'occupation coloniale au XIX ${ }^{\mathrm{e}}$ siècle est caractérisée par une stabilité relative. Cette période pacifique a favorisé l'éclosion des premiers lieux habités pour donner naissance aux lieux habités de la période coloniale (Figure 2). Il s'agit des localités de Assaba, Akatakou, Gotcha, Alétan, Okouta-Ossé, Kagourè, OkoutaAdjaba pour le peuple Isha au nord et les localités de Gbèdjè, Kafégnigbé, Montèwo, Idjou, Alé, Kamala, Abidji pour les Ifè au sud. $\mathrm{Au}$ cours de cette période, on note la pénétration de l'aire culturelle Isha-Ifè par les premiers immigrants dahoméens. Ce qui a donné naissance à des localités de Odjogbilè, Krabahou, Clougbou et Kadjogbé. Par ailleurs, les peuples Isha-Ifè ont connu durant cette période, à l'instar des peuples voisins Mahi, Idaasha et Sabè, le départ des bras valides en direction des pays de la sous-région notamment, le Ghana et la Côte-d'Ivoire à la recherche du bien-être (Akobi, 1997).

La troisième période, la moins longue, est celle postcoloniale, caractérisée par d'importants flux migratoires. On note dans cette période l'arrivée de nombreux immigrants du sud et du nord Bénin, d'une part ; le retour dans les années 60 des émigrés rapatriés du Ghana et de la Côte-d'Ivoire et aussi le départ massif des autochtones en direction du Nigéria au début des années 80 , d'autre part. Cette période postcoloniale est celle au cours de laquelle il y a eu plus de lieux habités créés comme l'indique la Figure 2 selon les investigations. Les résultats des enquêtes de terrain ont permis de réaliser la courbe de l'évolution de la création des lieux habités (Figure 3).

Cette évolution, quoique progressive, a été lente depuis les origines jusqu'à la fin du $\mathrm{XX}^{\mathrm{e}}$ siècle. La tendance progressive actuelle, plus remarquable, a débuté vers les années 70 avec une ampleur considérable à partir de 1990, comme le montre l'inflexion de la courbe. La Commune compte aujourd'hui plus de deux cent trente (230) lieux habités à plein temps, composés de villages, hameaux, fermes et campements peuhl.

En effet, cette dernière période est celle de l'éclosion de nombreuses fermes créées aussi bien par les autochtones que par les allochtones, surtout à partir des années 1990. En fait, depuis quelques décennies, la Commune de Bantè, à l'instar des autres Communes du Moyen-Bénin, reçoit d'importantes vagues de migrants des groupes socioculturels venus du sud (Adja, Fon, Holli...), du centre (Idaasha, Mahi...), du nord Bénin (Lokpa, Otamari, Pila-pila...) et même des pays voisins (Kotokoli, Kabyè, 
Peulh). Ces migrants qui s'adonnent pour la plupart aux activités agropastorales créent des fermes et campements ou cohabitent avec les autochtones. On peut citer des fermes et hameaux tels que Gando, Elamèta, Adja-Pira, Otinkouagni, Fomon, Soula, Aguélé, Fèro-Iya, Ouaké, Edahoué, Péhoudiè, Afrance, Akando (Figure 2). Par ailleurs, depuis quelques années, ce sont les Ifè du sud de Bantè qui sont contraints par la rareté de terres cultivables à migrer vers le nord-est de la Commune de Bantè, précisément dans la zone de Djagbalo, pour créer de nouvelles fermes de cultures (Akobi, 1997).

De ce qui précède, on peut dire que le peuplement de la Commune de Bantè est marqué par un long processus mais dynamique sur trois grandes périodes de durée et d'intensité inégales. Il a abouti à une dissémination des lieux habités sur l'ensemble de son territoire à la faveur d'une colonisation agricole spontanée.

Malgré ces flux migratoires, la densité de la population de la Commune est toujours relativement faible avec 40 habitants $/ \mathrm{km}^{2}$; une densité inférieure à la moyenne nationale qui est de 50 habitants $/ \mathrm{km}^{2}$ (INSAE, 2016). Ce peuple qui est à plus de $75 \%$ agricole, exploite les ressources naturelles disponibles; ce qui n'est pas sans conséquence sur l'évolution du couvert végétal.

\section{Exploitation des ressources naturelles, un déterminant de l'évolution du couvert végétal}

L'exploitation et l'utilisation des ressources naturelles par les hommes sont les principaux facteurs de l'évolution du couvert végétal d'un milieu. Plus de $75 \%$ de la population de la Commune de Bantè exercent des activités agraires. Ces dernières que sont l'agriculture, l'élevage, la chasse, les exploitations forestières et la pêche, consistent à l'exploitation des ressources naturelles, notamment le sol, l'eau et la végétation. Les plus intenses de ces activités qui nécessitent d'être mises en exergue sont l'agriculture, l'exploitation forestière et la carbonisation.

L'agriculture pratiquée est extensive et la technique culturale repose sur l'itinérance sur brûlis avec l'incinération systématique des arbres.

L'exploitation forestière à grande échelle a commencé dans la Commune de Bantè dans les années 1960 et 1970 avec l'implantation de trois scieries modernes à Bantè, Pira et Malomi. Cette exploitation forestière a été marquée par la destruction des formations naturelles y compris la forêt classée d'Agoua et l'abattage systématique des grands arbres tels que Pterocarpus erinaceus, Milicia excelsa, Khaya senegalensis. Quant à la carbonisation qui a démarré avec les épouses des colons agricoles dans la décennie 1980, elle a fini par prendre de l'ampleur suite à la forte demande des populations des centres urbains de charbons. Ces activités qui déterminent la dynamique de l'occupation spatiale, contribuent sans doute à l'évolution du couvert végétal.

\section{Evolution du couvert végétal de la commune de Bantè en 1986, 2000 et 2016}

En 1986, la physionomie de la végétation dans la Commune de Bantè était dominée par les savanes arborées et arbustives et les forêts claires et savanes boisées, qui représentaient respectivement $63,80 \%$ et $24,76 \%$ de la superficie. Les forêts denses sèches étaient présentes et occupaient 3,29\% des superficies. Les forêts galeries, y étaient également rencontrées $(1,70 \%)$ et étaient présentes le long des cours d'eau. Les mosaïques de champs et de jachères et les agglomérations occupaient respectivement $6,19 \%$ et $0,15 \%$ de la superficie de la Commune (Figure 4). Les autres unités (savane saxicoles et plantations) étaient également présentes mais en très faibles proportions.

En 2000, la végétation du secteur d'étude était dominée par les savanes arborées et arbustives $(65,45 \%)$ et les forêts claires et savanes boisées $(24,24 \%)$ et se retrouvent un peu partout dans la Commune. On retrouve également les autres formations végétales naturelles notamment les forêts claires et savanes boisées $(1,65 \%)$, les forêts galeries $(1,58 \%)$. Les savanes saxicoles qui ne représentent que $0,02 \%$ tendent à disparaître. Les mosaïques de champs et jachères $(6,68 \%)$ 
et les agglomérations $(0,22 \%)$ sont aussi rencontrés (Figure 5).

La physionomie de la végétation dans la Commune de Bantè en 2016 était dominée par les savanes arborées et arbustives qui représentaient 52,39\% de la superficie. Les autres formations végétales naturelles notamment les forêts claires et savanes boisées, les forêts denses sèches et les forêts galeries étaient également présentes et occupent respectivement $11,14 \%, 1,44 \%$ et $1,49 \%$ des superficies tandis que les savanes saxicoles qui étaient présentes en 1986 et 2000, ont complètement disparu. Les formations anthropiques telles que les mosaïques de champs et jachères et les plantations occupent respectivement $21,55 \%$ et $11,6 \%$ alors que les agglomérations $0,36 \%$ de la superficie de la Commune (Figure 6). Les affleurements rocheux qui étaient absents en 1986 et en 1995 sont apparus mais en très faibles proportions.

\section{Dynamique des unités paysagères dans la commune de Bantè}

$\checkmark$ Dynamique des unités paysagères de la commune entre 1986 et 2000

La dynamique des unités d'occupation du sol dans la Commune de Bantè de 1986 à 2000 est résumée par la matrice de transition (Tableau 1).

Dans les cellules des lignes et des colonnes se trouvent respectivement les unités d'occupation du sol de 1986 et de 2000. Les conversions se font des lignes vers les colonnes. Les cellules de la diagonale correspondent aux superficies des unités qui sont demeurées stables de 1986 à 2000. Les unités qui sont en dehors de la diagonale représentent les changements des unités d'occupation du sol.

L'examen de la matrice de transition révèle que toutes les unités d'occupation du sol observées en 1986 sont également présentes en 2000. Mais, de façon générale, on remarque que les formations naturelles ont subi deux modes de conversion probable : la savanisation et/ou l'anthropisation.

De 1986 à 2000, les forêts galeries ont connu une diminution de leur superficie qui est passée de $47,4 \mathrm{~km}^{2}$ à $44,03 \mathrm{~km}^{2}$, soit un taux de régression de $0,12 \%$ avec un taux de conversion de 5,55\%. L'analyse de la matrice de transition montre que $2,63 \mathrm{~km}^{2}$ des forêts galeries ont été converties en champs et jachères. Les forêts galeries connaissent une évolution essentiellement régressive au profit des formations anthropisées. Cette régression des forêts galeries est due à l'exploitation forestière et aux activités agricoles pratiquées dans ces formations.

Les forêts denses sèches ont connu aussi une diminution de leur superficie qui est passée de 91,82 $\mathrm{km}^{2}$ en 1986 à 46,03 km² en 2000 soit un taux de régression de $1,64 \%$ et un taux de conversion de 53,29\%. En effet, l'examen de la matrice de transition montre que respectivement $30,74 \mathrm{~km}^{2}, 15,96 \mathrm{~km}^{2}$ et $2,23 \mathrm{~km}^{2}$ des forêts denses sèches se sont converties en forêts claires et savanes boisées, en savanes arborées et arbustives et en champs et jachères. La régression des forêts denses sèches est due à la fois à l'exploitation forestière et aux activités agricoles pratiquées dans ces formations.

Les forêts claires et savanes boisées ont également connu une diminution de leur superficie de 1986 à 2000. Elles sont passées de $690,73 \mathrm{~km}^{2}$ à $676,39 \mathrm{~km}^{2}$ avec un taux annuel de régression de $0,51 \%$. L'analyse de la matrice de transition montre que respectivement $3,14 \mathrm{~km}^{2}, 41,49 \mathrm{~km}^{2}$ et 5,53 $\mathrm{km}^{2}$ des forêts claires et savanes boisées ont été converties en forêts denses sèches, en savanes arborées et arbustives et en mosaïques champs et jachères; soit un taux de conversion de $7,26 \%$. Cette régression des forêts claires et savanes boisées est due essentiellement aux activités agricoles et à l'exploitation forestière.

De même, les savanes saxicoles ont connu une diminution de leur superficie qui est passée de $1,49 \mathrm{~km}^{2}$ en 1986 à $0,5 \mathrm{~km}^{2}$ en 2000 ; soit un taux de régression de $0,04 \%$. Une partie de cette formation $\left(0,99 \mathrm{~km}^{2}\right)$ s'est convertie en champs et jachères, soit un taux de conversion de $66,27 \%$. Cette régression est due essentiellement aux activités agricoles. Avec cette tendance, cette formation risque de disparaître.

Par contre, les savanes arborées et arbustives ont connu une augmentation de leur 
superficie de 1986 à 2000 avec un taux annuel de progression de 1,65\%. En effet, leur superficie est passée de $1780,07 \mathrm{~km}^{2}$ en 1986 à $1826,13 \mathrm{~km}^{2}$ en 2000 . Cette tendance est due en grande partie à la conversion des savanes arborées et arbustives en mosaïques de champs et jachères. En effet, l'analyse de la matrice de transition révèle que $6,32 \mathrm{Km}^{2}$ des savanes arborées et arbustives ont été transformées en mosaïques de champs et jachères et $5,08 \mathrm{Km}^{2}$ en forêts claires et savanes boisées. Le taux de conversion est de $0,64 \%$ montrant ainsi une relative stabilité de ces formations végétales.

Quant aux mosaïques de champs et jachères, elles ont connu une extension. Leur superficie est passée de 172,65 km² en 1986 à $186,25 \mathrm{~km}^{2}$ en 2000 . Cette extension est due à l'accroissement de la population. Le taux de progression est de 0,49\%. L'examen de la matrice de transition montre que respectivement $2,09 \mathrm{~km}^{2}, 2 \mathrm{~km}^{2}$ et seulement $0,01 \mathrm{~km}^{2}$ des mosaïques de champs et jachères ont été converties en plantations, en agglomérations et savanes arborées et arbustives; soit un taux de conversion de 2,38\%.

Par ailleurs, les plantations et les agglomérations ont connu une légère augmentation de leur superficie avec un taux de progression respectif de $0,08 \%$ et $0,07 \%$.

\section{Dynamique des unités paysagères de la commune entre 2000 et 2016}

La matrice de transition (Tableau 2) calculée entre 2000 et 2016 permet de mieux apprécier la dynamique des formations végétales et des autres unités d'occupation du sol dans la Commune de Bantè.

L'examen de la matrice de transition (Tableau 2) révèle qu'en dehors des savanes saxicoles, les unités d'occupation du sol observées en 2000 sont également présentes en 2016. On note aussi une apparition des affleurements rocheux. Mais, de façon générale, on remarque que les formations naturelles ont subi un seul mode de conversion probable: l'anthropisation. Les taux de conversion (Tableau 2) permettent une meilleure compréhension des conversions subies par les différentes unités paysagères de 2000 à 2016.

De 2000 à 2016, les forêts galeries ont connu une diminution de leur superficie qui est passée de $44,03 \mathrm{~km}^{2}$ à $41,60 \mathrm{~km}^{2}$, soit un taux de régression de $0,09 \%$. L'analyse de la matrice de transition montre que $2,43 \mathrm{~km}^{2}$ des forêts galeries ont été converties en champs et jachères, soit un taux de conversion de 5,52\%.

Les forêts denses sèches ont connu également une diminution de leur superficie qui est passée de 46,03 km² en 2000 à 41,620 $\mathrm{km}^{2}$ en 2016 ; soit un taux de régression de $0,21 \%$ et un de conversion de $87,91 \%$. L'analyse de la matrice de transition montre que respectivement $14,56 \mathrm{~km}^{2}, 20,66 \mathrm{~km}^{2}$ et $5,24 \mathrm{~km}^{2}$ des forêts denses sèches se sont converties en forêts claires et savanes boisées, en savanes arborées et arbustives et en champs et jachères. La régression des forêts denses sèches est due essentiellement à l'exploitation forestière et aux activités agricoles pratiquées dans ces formations. Le fort taux observé au niveau de ces formations témoigne qu'elles sont très perturbées avec l'arrivée massive des colons agricoles dans la commune.

De 2000 à 2016, les forêts claires et savanes boisées ont également connu une diminution de leur superficie qui est passée de $676,39 \mathrm{~km}^{2}$ à $310,81 \mathrm{~km}^{2}$ avec un taux de régression de $13,10 \%$. L'analyse de la matrice de transition montre qu'une grande partie des forêts claires et savanes boisées a été convertie en forêts denses sèches, en savanes arborées et arbustives, en plantation et en mosaïques champs et jachères ; soit un taux de conversion de $81,4 \%$. Cette régression des forêts claires et savanes boisées est due essentiellement aux activités agricoles et à l'exploitation forestière.

Les savanes arborées et arbustives ont connu une évolution régressive de leur superficie passant de $1826,13 \mathrm{~km}^{2}$ en 2000 à $1780,07 \mathrm{~km}^{2}$ en 2016 avec un taux de progression de $13,07 \%$. Cette tendance est due en grande partie à la conversion des savanes arborées et arbustives en forêts denses sèches, en savanes arborées et arbustives, en plantation et en mosaïques champs et jachères. Le taux de conversion est de $38,17 \%$. La régression des savanes arborées et arbustives 
est due aux activités agricoles et à l'exploitation forestière. Par contre, les savanes saxicoles qui étaient présentes en 1986 et en 2000 ont complètement disparu. En effet, respectivement $0,22 \mathrm{~km}^{2}$ et $0,29 \mathrm{~km}^{2}$ des savanes saxicoles se sont converties en champs et jachères et en affleurement rocheux ; soit un taux de conversion de $100 \%$.

Quant aux mosaïques de champs et jachères et les plantations, elles ont connu une très grande extension. Leurs superficies sont passées respectivement de $186,25 \mathrm{~km}^{2}$ et 4,14 $\mathrm{km}^{2}$ en 2000 à $601,31 \mathrm{~km}^{2}$ et $323,6 \mathrm{~km}^{2}$ en 2016 ; soit un taux de progression respectif de $14,88 \%$ et $11,45 \%$. Cette extension est due aux activités agricoles et à l'intérêt qu'accordent les populations de la Commune de Bantè à la filière anacarde. L'examen de la matrice de transition montre qu'une grande partie des mosaïques de champs et jachères ont été converties en plantations $\left(95,48 \mathrm{~km}^{2}\right)$, en savanes arborées et arbustives $\left(32,05 \mathrm{~km}^{2}\right)$ et seulement $3,46 \mathrm{~km}^{2}$ en agglomérations.

En ce qui concerne les agglomérations, elles ont connu une augmentation de leur superficie qui est passée de $6,16 \mathrm{~km}^{2}$ en 2000 à $10,18 \mathrm{~km}^{2}$ en 2016; soit un taux de progression de $0,14 \%$. Leur taux de conversion est nul.

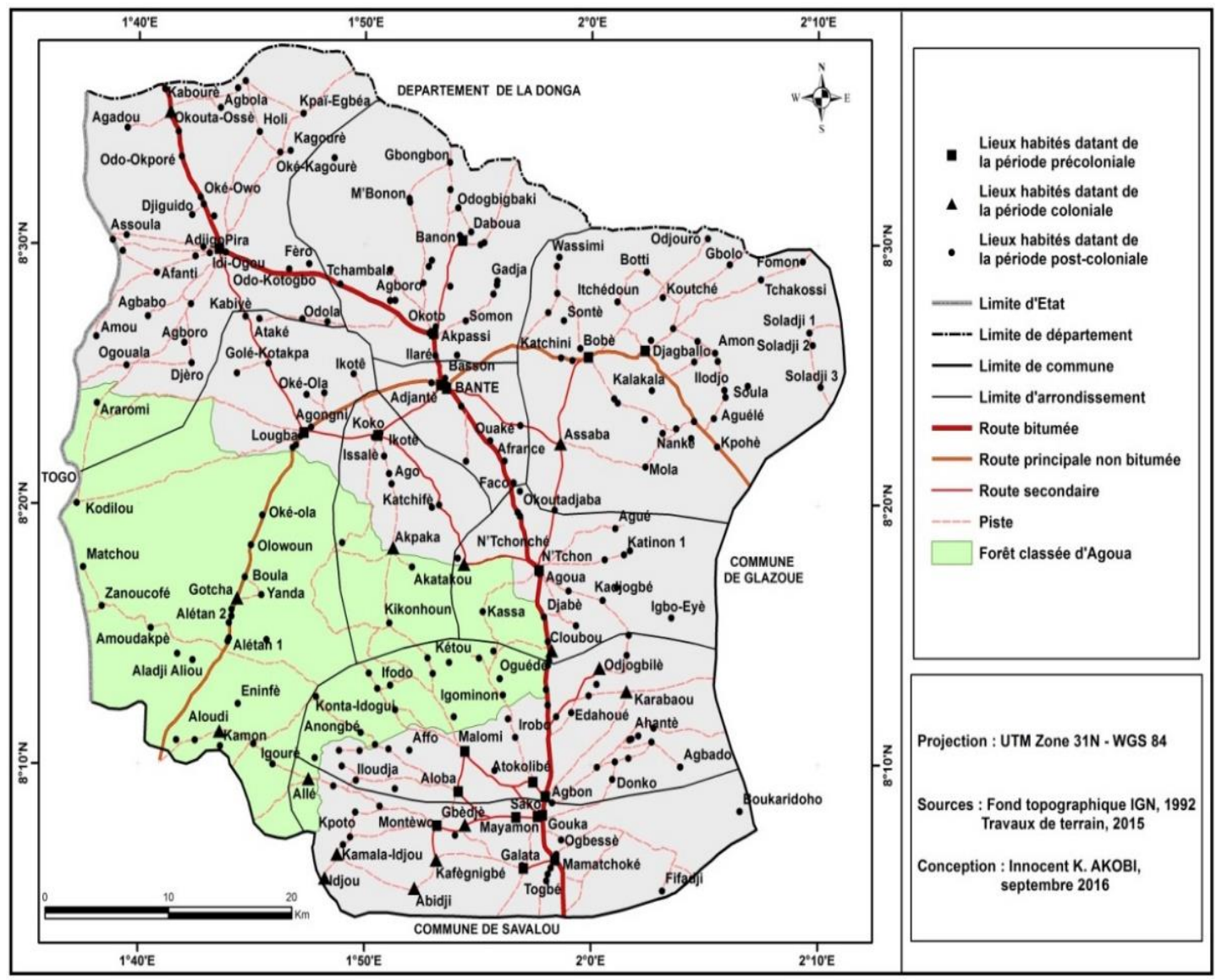

Figure 2: La dynamique du peuplement de la Commune de Bantè. Enquête de terrain Mars 2016 - juillet 2017. 


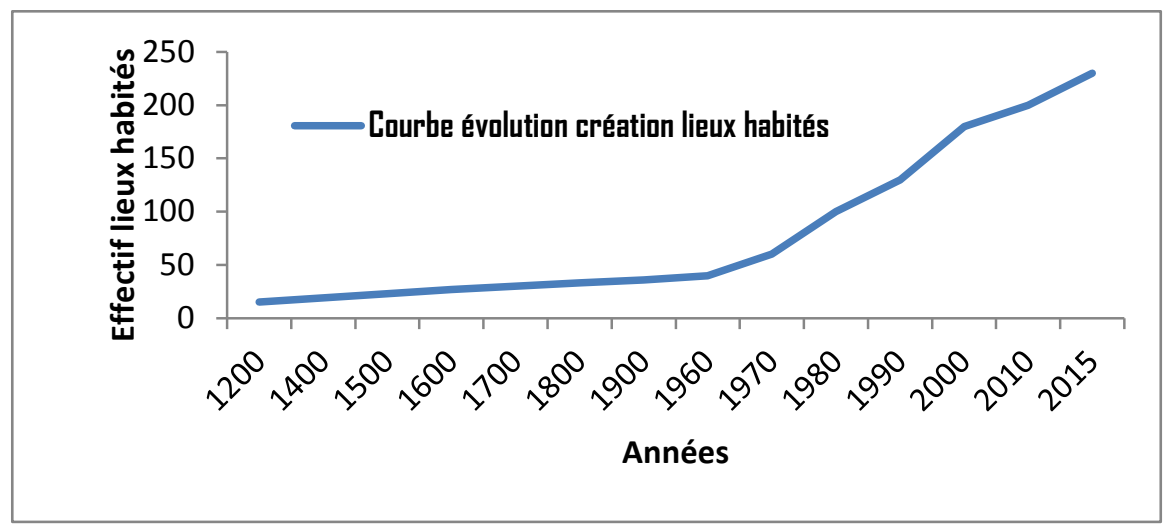

Figure 3: Evolution de la création des lieux habités de la Commune de Bantè des origines à nos jours. Adam et Boko (1983), Akobi (1997) et enquêtes de terrain 2014-2016.

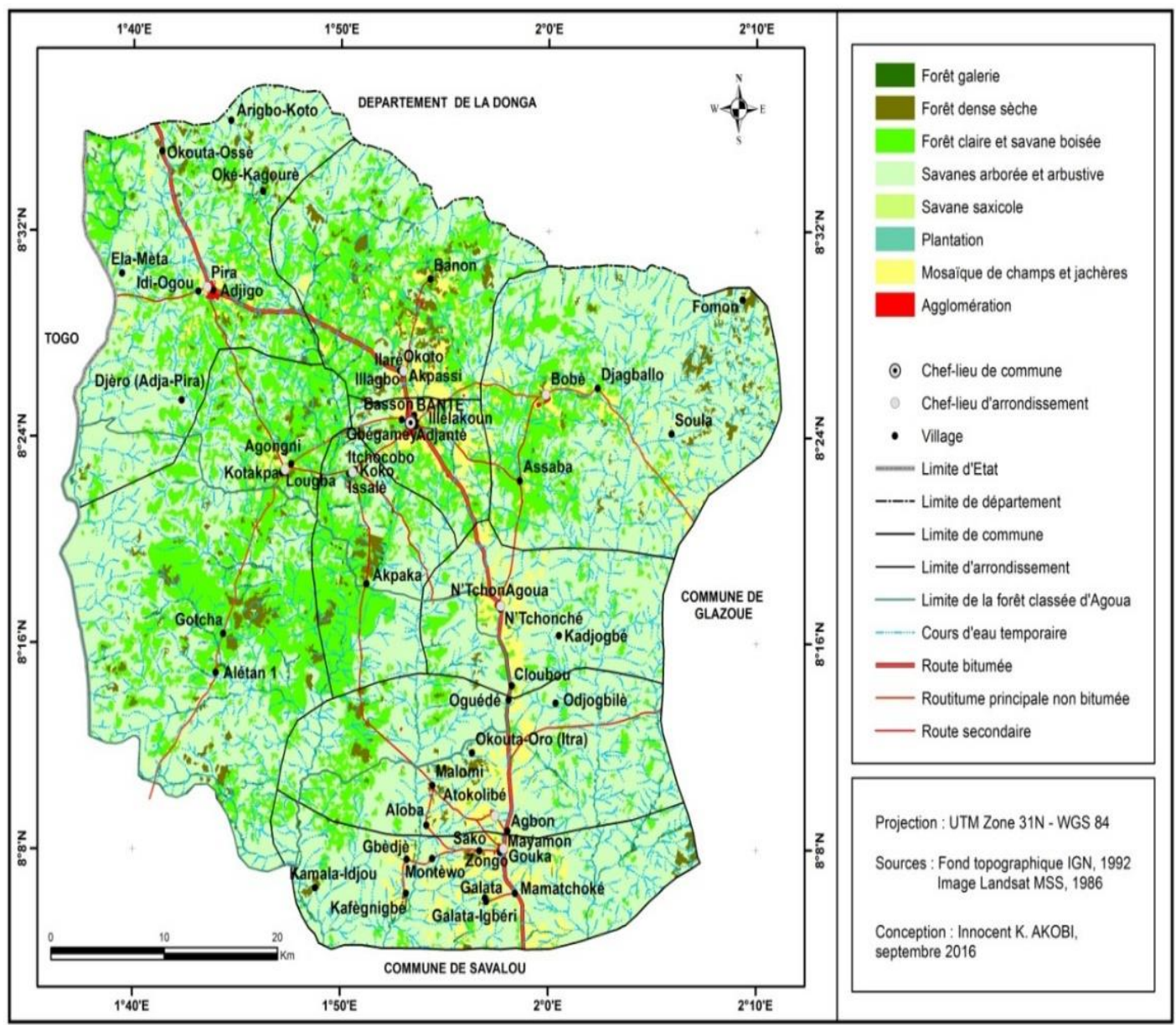

Figure 4: Formations végétales et autres unités d'occupation du sol dans la commune de Bantè en 1986. Interprétation des images Landsat TM de 1986. 


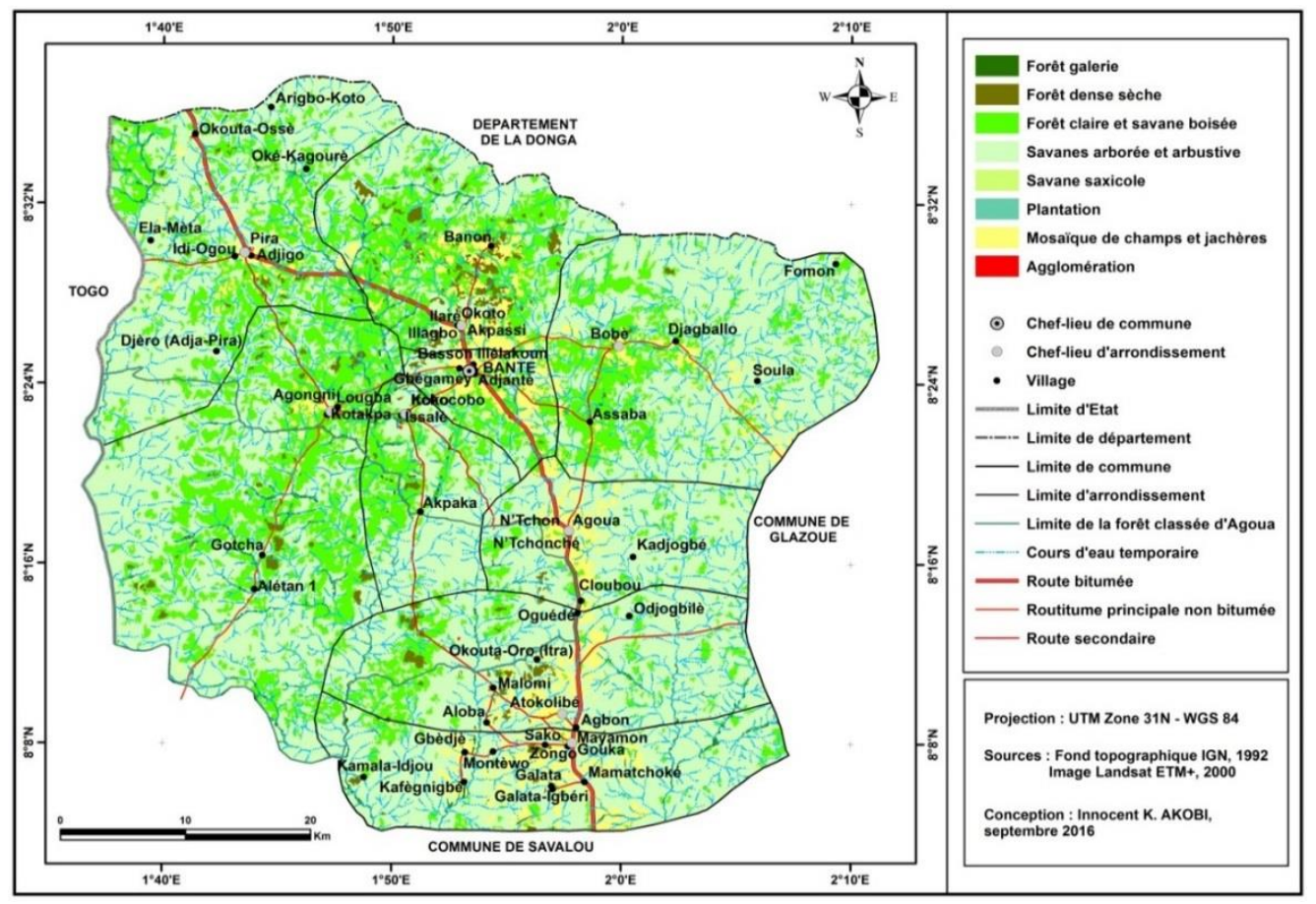

Figure 5: Formations végétales et autres unités d'occupation du sol dans la commune de Bantè en 2000. Interprétation des images Landsat TM de 2000.

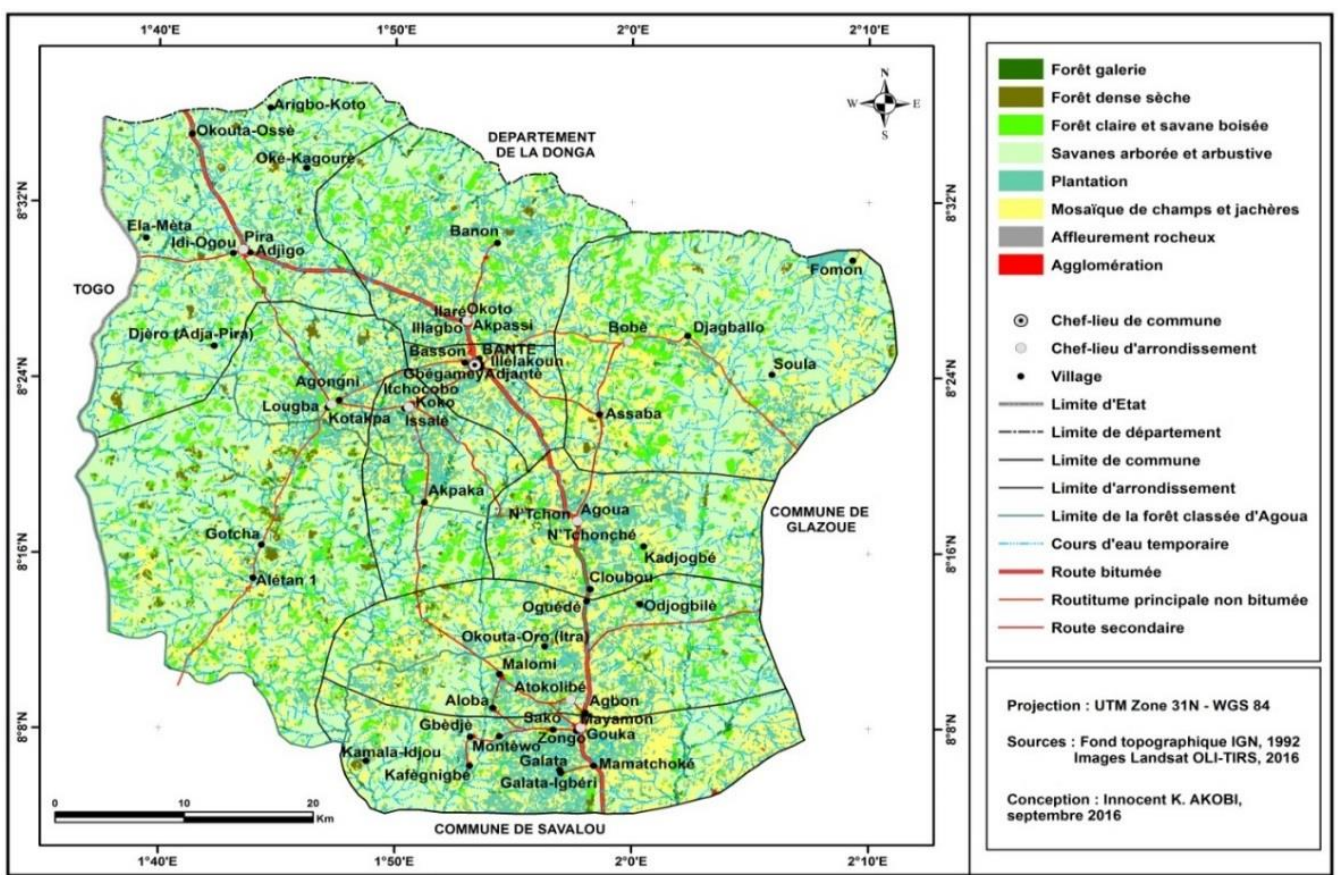

Figure 6: Formations végétales et autres unités d'occupation du sol dans la commune de Bantè en 2016. Interprétation des images Landsat ETM+ de 2016. 
Tableau 1: Matrice de transition de l'occupation du sol entre 1986 et 2000.

\begin{tabular}{lccccccccc}
\hline \multirow{2}{*}{ Unités de 1986 } & \multicolumn{8}{c}{ Unités de 2000 } & \\
\cline { 2 - 10 } & FG & FDS & FCSB & SASA & SS & PL & MCJ & AG & $\begin{array}{c}\text { Sup. totale en } \\
\mathbf{1 9 8 6}\left(\mathbf{K m}^{2}\right)\end{array}$ \\
\hline FG & 44,77 & 0 & 0 & 0 & 0 & 0 & 2,63 & 0 & 47,40 \\
FDS & 0 & 42,89 & 30,74 & 15,96 & 0 & 0 & 2,23 & 0 & 91,82 \\
FCSB & 0 & 3,14 & 640,57 & 41,49 & 0 & 0 & 5,53 & 0 & 690,73 \\
SASA & 0 & 0 & 5,08 & 1768,67 & 0 & 0 & 6,32 & 0 & 1780,07 \\
SS & 0 & 0 & 0 & 0 & 0,50 & 0 & 0,99 & 0 & 1,49 \\
PL & 0 & 0 & 0 & 0 & 0 & 2,04 & 0 & 0 & 2,04 \\
MCJ & 0 & 0 & 0 & 0,01 & 0 & 2,09 & 168,55 & 2,00 & 172,65 \\
AG & 0 & 0 & 0 & 0 & 0 & 0 & 0 & 4,16 & 4,16 \\
Sup. totale en & 44,77 & 46,03 & 676,39 & 1826,13 & 0,50 & 4,14 & 186,25 & 6,16 & $\mathbf{2 7 9 0}$ \\
$2000\left(\mathrm{Km}^{2}\right)$ & & & & & & & & & \\
\hline
\end{tabular}

FG : Forêt galerie ; FCSB : Forêt claire et savane boisée ; SASA : Savanes arborée et arbustive ; SS : Savane saxicole ; PL : Plantation; CJ : Mosaïques de Champs et jachères ; AG : Agglomération. Sup. : Superficie ; 44,77: Superficie demeurée stable entre 1986 et 2000.

Tableau 2: Matrice de transition de l'occupation du sol entre 2000 et 2016.

\begin{tabular}{|c|c|c|c|c|c|c|c|c|c|}
\hline \multirow{2}{*}{ Unités de 2000} & \multicolumn{8}{|c|}{ Unités de 2016} & \multirow{2}{*}{$\begin{array}{c}\text { Sup. totale en } \\
2000\left(\mathbf{k m}^{2}\right)\end{array}$} \\
\hline & FG & FDS & FCSB & SASA & PL & MCJ & $\mathbf{A R}$ & AG & \\
\hline FG & 41,60 & 0 & 0 & 0 & 0 & 2,43 & 0 & 0 & 44,03 \\
\hline FDS & 0 & 5,57 & 14,56 & 20,66 & 0 & 5,24 & 0 & 0 & 46,03 \\
\hline FCSB & 0 & 24,95 & 125,83 & 279,81 & 85,954 & 159,798 & 0 & 0,05 & 676,39 \\
\hline SASA & 0 & 9,74 & 170,42 & 1129,08 & 139,26 & 377,26 & 0 & 0,38 & 1826,13 \\
\hline SS & 0 & 0 & 0 & 0 & 0 & 0,22 & 0,29 & 0 & 0,50 \\
\hline PL & 0 & 0 & 0 & 0 & 2,90 & 1,11 & 0 & 0,13 & 4,14 \\
\hline $\mathrm{MCJ}$ & 0 & 0 & 0 & 32,05 & 95,48 & 55,26 & 0 & 3,46 & 186,25 \\
\hline $\mathrm{AG}$ & 0 & 0 & 0 & 0 & 0 & 0 & 0 & 6,16 & 6,16 \\
\hline $\begin{array}{l}\text { Sup. totale en } \\
2016\left(\mathrm{Km}^{2}\right)\end{array}$ & 41,60 & 40,25 & 310,81 & 1461,60 & 323,6 & 601,31 & 0,29 & 10,18 & 2790 \\
\hline
\end{tabular}

FG: Forêt galerie; FCSB : Forêt claire et savane boisée; SASA: Savanes arborée et arbustive ; SS: Savane saxicole; PL: Plantation; CJ: Mosaïques de Champs et jachères; AR: Affleurement rocheux ; AG: Agglomération. Sup.: Superficie 41,60: Superficie demeurée stable entre 2000 et 2016. 


\section{DISCUSSION}

L'évaluation de la pression anthropique sur le couvert végétal a révélé l'évolution régressive des formations végétales naturelles au profit des formations anthropiques. Ce processus régressif est marqué par la conversion des formations végétales allant jusqu'à la disparition totale de la forêt dense sèche au profit d'autres unité d'occupation du sol (forêts claires et savanes boisées, savanes arborées et arbustives, et champs et jachères) entre 1986 et 2016. Cette disparition serait certainement inhérente à la pression exercée sur cette formation végétale à des fins d'exploitation forestière durant de nombreuses années comme bois d'œuvre et bois de service (Pterocarpus erinaceus, Khaya senegalensis, Milicia excelsa). Des études réalisées sur des thématiques similaires ont montré que la conversion des formations végétales s'opère dans les unités spatiales dominées par des pressions exercées sur les ressources naturelles par le prélèvement des ligneux et l'agriculture (Tenté et al., 2011; Sitondji et al., 2013; Yabi et al., 2013; Avakoudjo et al., 2014).

Ainsi, les ligneux à gros diamètre présents au niveau des forêts denses sèches ont été exploités comme bois d'œuvre. Les autres formations végétales (forêts claires, savanes boisées, savanes arborées, savanes arbustives) n'ont pas totalement disparu mais ont tout de même connu une fragmentation au profit des mosaïques de champs et jachères, des plantations et des agglomérations. En 2016, les savanes arborées et arbustives constituent les formations végétales naturelles dominantes. Cela constitue un indicateur de dégradation car la végétation climacique à cette latitude est constituée par les forêts denses sèches, les forêts claires et les forêts galeries (Sitondji et al., 2006). La superficie des champs et jachères a connu une augmentation exponentielle. Cette augmentation exponentielle de la superficie des champs et jachères au détriment des formations végétales naturelles pourrait s'expliquer par la croissance démographique puisque la population de la Commune de
Bantè a pratiquement quadruplé dans l'espace de 34 ans. Carr et al. (2005) ont modélisé les facteurs déterminant une telle dynamique régressive des formations végétales. Ils sont parvenus à la conclusion que la régression des formations végétales et la déforestation sont les conséquences de la croissance démographique, des options de développement et des technologies utilisées. En examinant ces facteurs de façon approfondie, ces auteurs ont retenu que l'agriculture et l'exploitation forestière contribuent de façon déterminante à la dynamique régressive des formations végétales. Les travaux réalisés au Bénin et précisément dans le Département des Collines par Oloukoï et al. (2006) rejoignent les résultats de Carr et al. (2005) et permettent de justifier la dynamique régressive des formations végétales dans la Commune de Bantè par la pression anthropique.

\section{Conclusion}

L'étude de la pression anthropique sur le couvert végétal dans la Commune de Bantè révèle la tendance concurrente entre l'évolution spatio-temporelle de la végétation et du peuplement. Durant les trois dernières décennies, les formations anthropiques ont connu une augmentation de leur superficie au détriment des formations végétales naturelles. La gravité de cette menace ne doit pas laisser indifférentes les autorités. C'est pourquoi, la présente étude vise à attirer leur attention sur l'enjeu de la sauvegarde de l'environnement dans sa dimension végétale, surtout à l'ère du réchauffement planétaire. Des mesures de gestion plus efficace doivent être prises pour une planification de l'occupation spatiale rationnelle en vue de la durabilité du développement de la Commune.

\section{CONFLIT D'INTERETS}

Tous les auteurs déclarent à
l'unanimité qu'il n'existe aucun conflit
d'intérêts entre eux relatif à cet article.




\section{CONTRIBUTIONS DES AUTEURS}

KIA a initié la recherche, rédigé le protocole et conduit les enquêtes de terrain, s'est occupé de l'analyse historique des données sur l'occupation des terres et la rédaction de l'article. Il a également participé à l'analyse statistique des données et a écrit le manuscrit. EA a fait le traitement statistique et cartographique et l'interprétation des résultats, il a également fait la revue de littérature. IY a réalisé les cartes d'occupation du sol de la Commune, fait l'analyse, la mise en forme et la relecture du manuscrit. MB a donné des orientations méthodologiques, supervisé toutes les activités, contribué à la lecture et approfondi les analyses sur l'historique de l'évolution de la population.

\section{REFERENCES}

Akobi KI. 1997. Les migrations de population et leur impact socio-économique dans la Sous-préfecture de Bantè, mémoire de maîtrise de géographie, Université Nationale du Bénin, 135p.

Amani YC, Touré A. 2015. Implantations humaines et dégradation des forêts classées du Sud-Ouest de la Côte d'Ivoire : cas des Rapides Grah. TALOHA, Numéro 21, en ligne, consulté le 21 février 2018, http://www.taloha.info/document.php?id $=1397$.

Anthelme F, Mato MW, de Boissieu D, Giazzi F. 2006. Dégradation des ressources végétales au contact des activités humaines et perspectives de conservation dans le massif de l'Aïr (Sahara, Niger) », VertigO - la Revue Electronique en Sciences de l'Environnement, 7(2). [En ligne], DOI : 10.4000/vertigo.2224

Avakoudjo J, Mama A, Toko I, Kindomihou V, Sinsin B. 2014. Dynamique de l'occupation du sol dans le Parc National du W et sa périphérie au Nord-Ouest du
Bénin. Int. J. Biol. Chem. Sci., 8(6): 2608-2625.

Boko M, Niang I, Nyong A, Vogel C, Githeko A, Medany M, Osman-Elasha B, Tabo R, Yanda P. 2007. Climate Change 2007: Impacts, Adaptation and Vulnerability in Africa. Contribution of Working Group II to the Fourth Assessment Report of the Intergovernmental Panel on Climate Change. In Report of the Intergovernmental Panel on Climate Change, Parry ML, Canziani OF, Palutikof JP, Van Der Linden PJ, Hanson CE (eds). Cambridge University Press: Cambridge, UK; 433 - 467.

Carl LD, Suter L, Barberi A. 2005. Population dynamics and tropical deforestation: state of the debate and conceptual challenges. Population and Environment, 27(1): 89-113.

Houessou LG, Téka O, Toko I, Lykke AM, Sinsin B. 2013. Land use and land cover change at "W" Biosphere Reserve and its surroundings areas in Benin Republic (West Africa). Env. Nat. Res. Resear., 3(2): 87-101.

INSAE. 2016. Recensement Général de la Population et de l'Habitation (RGPH4). Résultats Définitifs. INSAE: Cotonou, Bénin ; 209.

Millogo D, Nikiema A, Koulibaly B, Zombre NP. 2017. Analyse de l'évolution de l'occupation des terres à partir de photographies aériennes de la localité de Loaga dans la province du Bam, Burkina Faso, Int. J. Biol. Chem. Sci., 11(5): 2133-2143.

Niassé M, Afouda A, Amani A. 2004. Réduire la Vulnérabilité de l'Afrique de l'Ouest aux Impacts du Climat sur les Ressources en Eau, les Zones Humides et la Désertification: Eléments de Stratégie Régionale de Préparation et 
d'Adaptation. UICN, Gland (Suisse) : Cambridge Royaume Uni ; 71.

Oloukoï J, Mama VJ, Agbo FB. 2006. Modélisation de la dynamique de l'occupation des terres dans le Département des Collines au Bénin. Télédétection, 6(4): 305-323.

RB (République du Bénin), 1999. Loi N $97-$ 029 du 15 janvier 1999 portant organisation des Communes en République du Bénin. Cotonou, Bénin, $15 \mathrm{p}$.

Tenté B, Baglo MA, Dossoumou J, Yèdomonhan H. 2011. Impacts des activités humaines sur les ressources forestières dans les terroirs villageois des communes de Glazoué et de DassaZoumè au centre-Bénin. Int. J. Biol. Chem. Sci., 5(5): 2022-2030

Sintondji LO, Agbossou EK, Degnissodé B. 2013. Dynamique de dégradation des forêts galeries et comblement du cours d'eau Agbado dans le département des Collines au Bénin. Int. J. Biol. Chem. Sci., 7(4): 1555-1567. DOI: http://dx.doi.org/10.4314/ijbcs.v7i4.12

Yabi I, Yabi BF, Dadegnon S. 2013. Diversité des espèces végétales au sein des agroforêts à base d'anacardier dans la commune de Savalou au Benin. International Journal of Biological and Chemical Sciences, 7: 696-706. 